\title{
Iatrogenic Lumbar Vertebral Fracture during Osteosynthesis for a Trochanteric Fracture of the Femur in Diffuse Idiopathic Skeletal Hyperostosis
}

\author{
Takeshi Sasagawa ${ }^{1}$, Hideki Murakami ${ }^{2}$, Yoshinobu Maruhashi ${ }^{1}$, Takeshi Segawa ${ }^{1}$, \\ Daiki Yamamoto ${ }^{1}$, Shusuke Shimizu ${ }^{1}$, Yasuhiko Morita ${ }^{1}$, Takuya Nakamura ${ }^{1}$ \\ ${ }^{I}$ Department of Orthopedics Surgery, Toyama Prefectural Central Hospital, Kanazawa University, Kanazawa, Japan \\ ${ }^{2}$ Department of Orthopaedic Surgery, Kanazawa University, Kanazawa, Japan
}

Vertebral fractures occur with only slight trauma in patients with diffuse idiopathic skeletal hyperostosis (DISH). However, a lumbar vertebra fracture, due to an intraoperative body position has not been previously reported. An 87-year-old woman with kyphosis sustained a left trochanteric fracture of her femur. The patient was placed in a supine position during the operation. Postoperatively, the patient experienced severe right thigh pain. Magnetic resonance imaging revealed an $L 4$ vertebral fracture. Computed tomography revealed ankylosis from the upper thoracic spine to the sacrum. While in a supine position under general anesthesia, the contact of the patient's lower back with operating table likely created a fulcrum at her lumbosacral spine acting as a long lever arm, bearing the mass of her upper body. We performed L1-S2 posterior stabilization. DISH patients with kyphosis placed in a supine position have an increased risk for lumbar vertebral fracture.

Keywords: Diffuse idiopathic skeletal hyperostosis; latrogenic fracture; Kyphosis; Lumbar vertebral fracture; Supine position

\section{Introduction}

Diffuse idiopathic skeletal hyperostosis (DISH) is a chronic disease characterized by calcification and ossification of spinal ligaments and peripheral enthuses [1]. Forty-nine to sixty-nine percent vertebral fractures in DISH occur secondary to minor trauma [2-6]. In the majority of cases, the mechanism of injury involved a fall from a standing or sitting position $[2,3]$. However, there are only a small number of reports on patients with ankylosing spinal disorders (ASD), whose mechanism of vertebral fracture involved an intraoperative body position. Our literature search revealed three such cases: two cases of ankylosing spondylitis [7] and one case of DISH [8]. The patients sustained a thoracic vertebral fracture during total hip arthroplasty in a supine position $[7,8]$. However, a lumbar vertebra fracture, due to an intraoperative body position in patients with DISH has not been previously reported.

We described the case of a kyphotic back patient with DISH who sustained a lumbar vertebral fracture during osteosynthesis for trochanteric fracture of the femur.

\section{Case Report}

An 87-year-old woman fell and sustained a right subtrochanteric fracture of the femur with displacement (Fig. 1A).

Received Dec 28, 2014; Accepted Dec 31, 2014

Corresponding author: Takeshi Sasagawa

Department of Orthopedics Surgery, Toyama Prefectural Central Hospital,

2-2-78 Nishinagae, Toyama 930-8550, Japan

Tel: +81-76-424-1531, Fax: +81-76-422-0667, E-mail: sasagawawagasasa@yahoo.co.jp 
The patient had a severe kyphotic lumbar curve with a marked lack of spinal mobility. We performed an operation to reduce the fracture with the patient in the lateral position (Fig. 1B). Postoperatively (Fig. 1C), the patient made satisfactory progress.

Seven months later, the patient fell again and sustained a left trochanteric fracture of the femur without displacement (Fig. 2A). Because reduction of the fracture was not needed, we performed the operation with the patient in supine position using a traction table. We tried to retain the severe kyphotic curvature in the patients back by piling cushions for support on the operating table while the patient was under general anesthesia. Fig. 2B showed the postoperative radiograph.

Postoperatively, the patient reported symptoms of severe right thigh pain. There was no redness or swelling at the surgical site of the prior right femoral subtrochanteric fracture, and radiographs revealed no abnormality. The
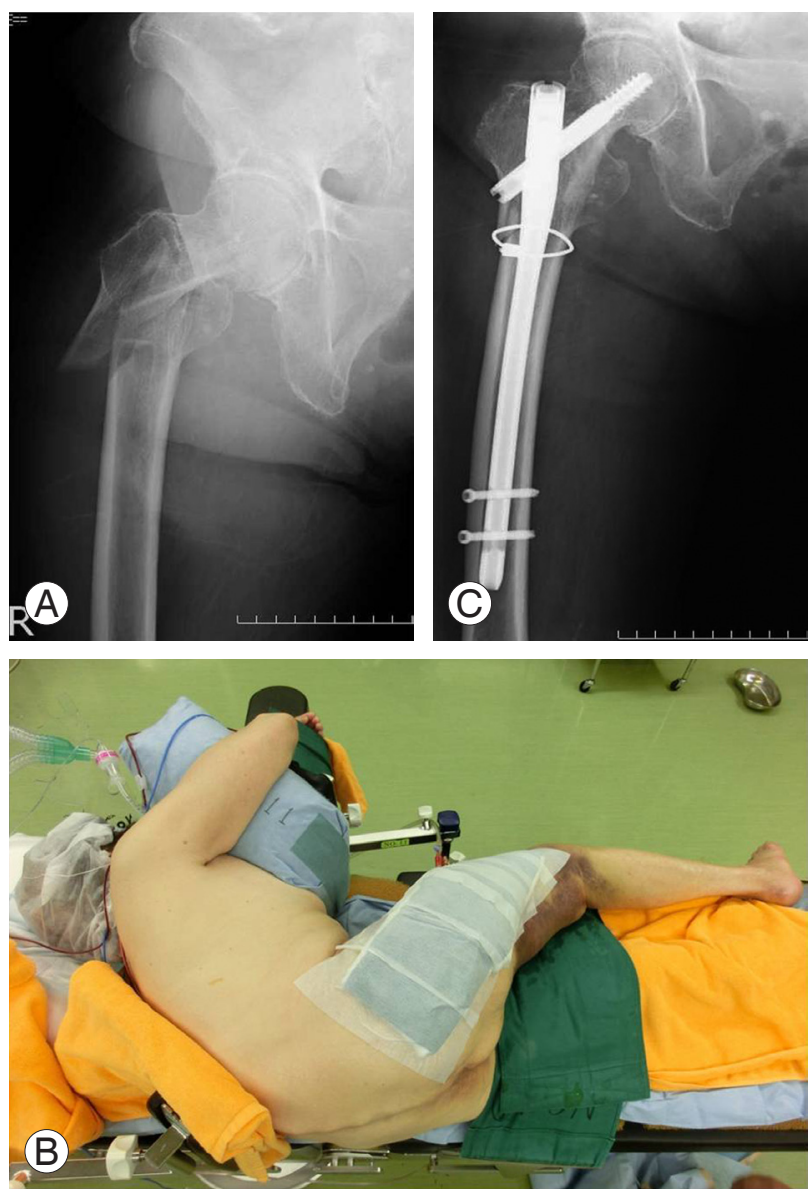

Fig. 1. (A) Preoperative radiograph of the right femur, (B) a photograph of the patient's lateral body position during surgery, and (C) a postoperative radiograph of the right femur. patient continued to experience right thigh pain, hence, one week after the osteosynthesis on left femur, the lumbar spine was evaluated by magnetic resonance imaging (MRI). MRI revealed an L4 vertebral fracture, right L4/5 foraminal stenosis, and a hematoma dorsal of the L5 spinous process. Therefore, the right thigh pain was diagnosed as radiculopathy of the right L4 nerve root (Fig. $3 \mathrm{~A})$. Computed tomography revealed ankylosis from her thoracic spine (T1) to sacrum due to ossification of the anterior longitudinal ligament (Fig. 3B). We diagnosed the case as DISH based on diagnostic criteria [9]. Due to the long lever arm created and the injury to both anterior and posterior elements of her vertebra, we performed L1S2 posterior stabilization using a pedicle screw from L1 to S1 and an S2 alar iliac screw while the patient was in a

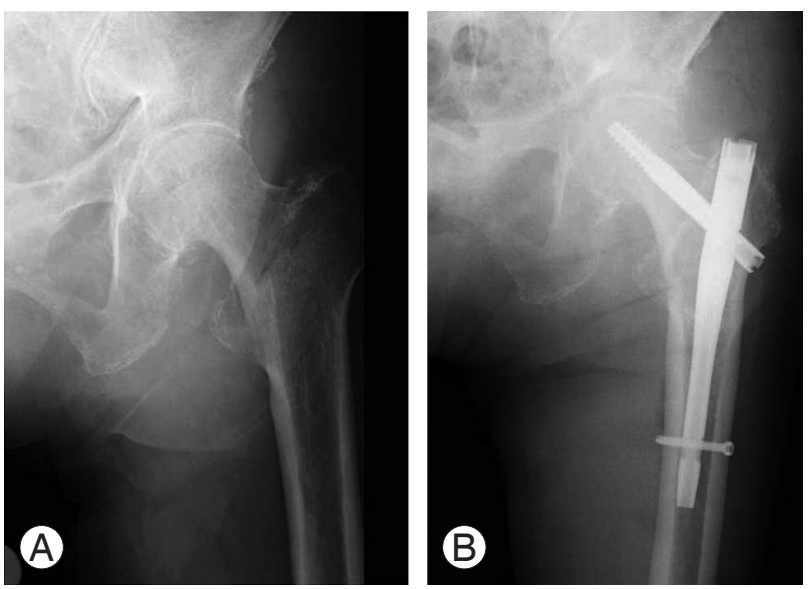

Fig. 2. (A) Preoperative radiograph of the left femur, and (B) a postoperative radiograph of the left femur.
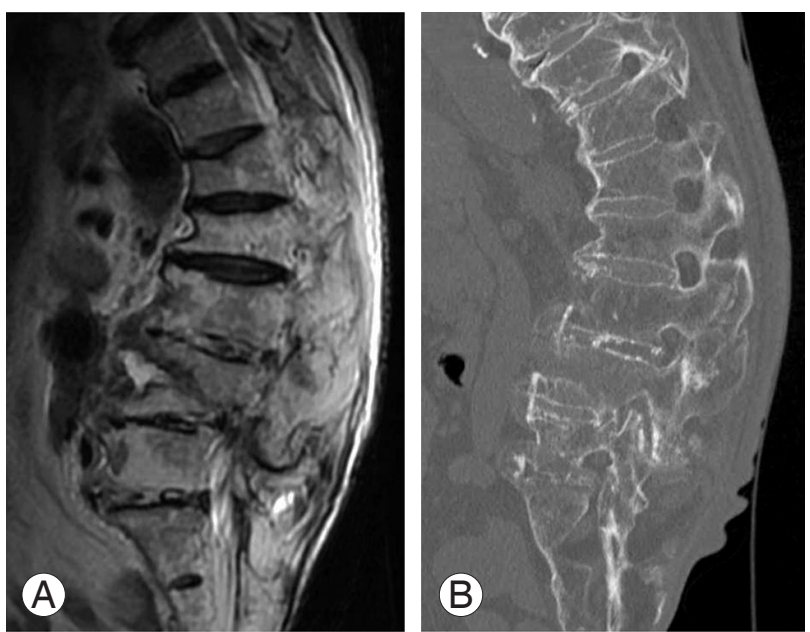

Fig. 3. (A) A T2-weighted magnetic resonance image of the lumbar spine, and (B) computed tomography of the lumbar spine. 
prone position (Fig. 4A). Postoperatively (Fig. 4B, C), the patient's right thigh pain resolved and she became ambulatory with a T-cane.

\section{Discussion}

Our patient underwent osteosynthesis in a supine position for a left proximal femoral fracture and developed an L4 vertebral fracture likely as a result of hyperextension. A hyperextension injury that precipitates a fracture is most commonly associated with vertebral fractures in ASD [3]. The arms and abdominal muscles would have likely provided some support had the patient been conscious when in the supine position. However, no support was possible while under general anesthesia, and a hyperextending force was likely applied to the spine. Although cushions were used for body support, they were compressed under her body weight and provided insufficient support.

The patient developed a fracture of the lower lumbar spine, intraoperatively. The cervical spine is the most common site for vertebral fractures in DISH patients, fol-

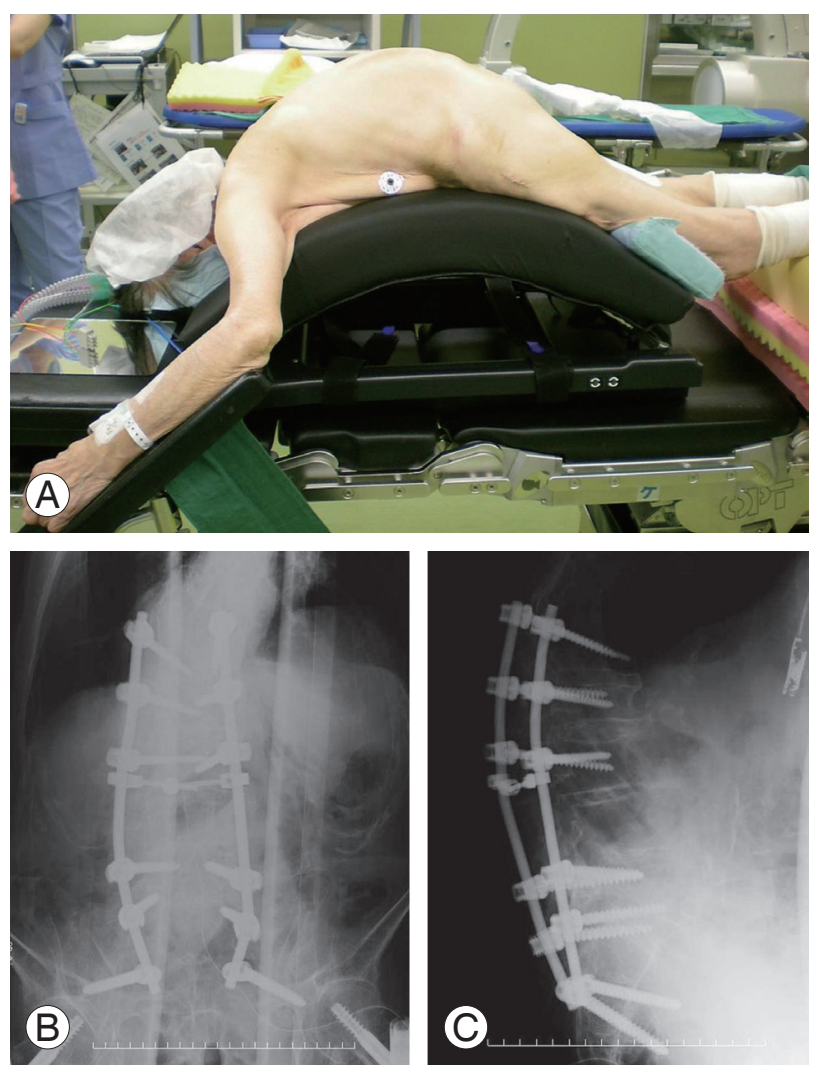

Fig. 4. (A) A photograph of the patient's body position during her second surgery, and $(\mathbf{B}, \mathbf{C})$ a postoperative radiograph of the lumbar spine. lowed by the thoracic and lumbar spine $[2,3,10]$. The fractures of the lower lumbar spine only account for a small number of cases $[2,3]$. Our patient had ankylosis and severe kyphosis from the upper $\mathrm{T} 1$ to the sacrum. Therefore, when the patient was in a supine position, her lower back region likely contacted the operating table creating a fulcrum at the lumbosacral spine as a long lever arm, bearing the mass of her upper body. A fracture is thought to have occurred at this site as a result.

An intraoperative vertebral fracture did not occur during prior surgery when the patient was in a lateral position, possibly because the same mechanical stress was not applied on the spine. Previously, it was reported that patients with ASD developed vertebral fractures due to a supine position during surgery for hip disease $[7,8]$. The patients in the previous reports also developed irreversible thoracic spinal cord injuries $[7,8]$. In our case, the patient only had radiculopathy but nevertheless required highly invasive lumbar spinal surgery. The lateral position should be selected when possible for surgery in patients with ASD.

\section{Conflict of Interest}

No potential conflict of interest relevant to this article was reported.

\section{References}

1. Forestier J, Rotes-Querol J. Senile ankylosing hyperostosis of the spine. Ann Rheum Dis 1950;9:321-30.

2. Caron T, Bransford R, Nguyen Q, Agel J, Chapman J, Bellabarba C. Spine fractures in patients with ankylosing spinal disorders. Spine (Phila Pa 1976) 2010;35:E458-64.

3. Westerveld LA, Verlaan JJ, Oner FC. Spinal fractures in patients with ankylosing spinal disorders: a systematic review of the literature on treatment, neurological status and complications. Eur Spine J 2009;18:145-56.

4. Paley D, Schwartz M, Cooper P, Harris WR, Levine AM. Fractures of the spine in diffuse idiopathic skeletal hyperostosis. Clin Orthop Relat Res 1991;(267): 22-32.

5. Wang YF, Teng MM, Chang CY, Wu HT, Wang ST. Imaging manifestations of spinal fractures in ankylosing spondylitis. AJNR Am J Neuroradiol 
2005;26:2067-76.

6. Belanger TA, Rowe DE. Diffuse idiopathic skeletal hyperostosis: musculoskeletal manifestations. J Am Acad Orthop Surg 2001;9:258-67.

7. Danish SF, Wilden JA, Schuster J. Iatrogenic paraplegia in 2 morbidly obese patients with ankylosing spondylitis undergoing total hip arthroplasty. J Neurosurg Spine 2008;8:80-3.

8. Königshausen M, Dudda M, Merle C, Schildhauer TA, Fehmer T. Thoracic vertebral body fracture after total hip replacement in diffuse idiopathic skeletal hyperostosis. Orthopedics 2012;35:e1000-4.

9. Resnick D, Niwayama G. Radiographic and pathologic features of spinal involvement in diffuse idiopathic skeletal hyperostosis (DISH). Radiology 1976;119:559-68.

10. Trent G, Armstrong GW, O’Neil J. Thoracolumbar fractures in ankylosing spondylitis. High-risk injuries. Clin Orthop Relat Res 1988;227:61-6. 